[pp.99-120]

Vol. 4(1), Enero - Junio, 2016

http://dx.doi.org/10.15359/rnh.4-1.8

\title{
Experiencias universitarias
} inter-generacionales en proyectos de participación comunitaria: construyendo aprendizajes significativos para la vida y lazos de solidaridad generacional

Intergenerational University Experiences in Community Participation Projects: Building Generational Solidarity Links and Significant Learning for Life

Ana Monge Barrantes

Universidad Nacional Costa Rica analuzmonge@yahoo.com

\section{Resumen}

El presente artículo relata los inicios y las experiencias generadas en dos actividades de docencia y extensión. La primera se trata de los cursos de "Cultura y persona adulta mayor: retos del nuevo milenio"; la segunda, titulada "Educando para la recreación y uso del tiempo Libre". El eje central del trabajo de extensión y docencia ha sido el diálogo intergeneracional en la formulación y ejecución de proyectos que coadyuven a la construcción de aprendizajes significativos para la vida. En los encuentros intergeneracionales participan estudiantes de la Universidad Nacional que asisten al Centro de Estudios Generales y personas adultas mayores provenientes de varias 
comunidades heredianas localizadas en centros diurnos y hogares. Estas actividades se realizan mediante la formulación y ejecución de programas intergeneraciones comprendidos en el marco de la acción institucional que interconecta la extensión universitaria con la docencia. Después de varios años de realizar estas labores en el marco del Proyecto UNA-Vida, se desea compartir y dar a conocer esta experiencia, cuyos resultados han sido muy satisfactorios y gratificantes; lo que reafirma el compromiso de seguir trabajando en la consecución de aprendizajes significativos para la vida que posibiliten el fortalecimiento de la acción sustantiva universitaria para un nuevo humanismo en el siglo XXI.

Palabras clave: personas Adultas mayores, estudiantes universitarios, programas intergeneracionales, proyectos recreativos, diálogos intergeneracionales, aprendizajes significativos para la vida, nuevo humanismo.

\begin{abstract}
This article reports the beginnings and experiences brought about in two teaching and extension activities. The first activity related to courses titled "Culture and older people: new millennium challenges". The second one was titled "Educating for recreation and use of free time". The extension and teaching activities focused on intergenerational dialog to design and execute projects contributing to building significant learning for life. Students from the National University of Costa Rica attended these intergenerational meetings; they study at the General Studies Center of this university. The older people who also attended the meetings came from different Heredia communities; they frequent elder day care centers and nursing homes. These activities are accomplished through the designing and execution of intergenerational programs within the institutional action framework that connects university extension and teaching. After years of carrying out tasks within the "UNA-Vida" project, this experience whose results have been very satisfactory and rewarding deserves to be shared and publicized. This reaffirms the commitment to continue working in the achievement of significant learning for life to enable strengthening of the substantive university action for a new humanism in the 21 rst century.
\end{abstract}

Keywords: Older people, university students, intergenerational programs, recreational projects, intergenerational dialogs, significant learning for life, new humanism. 


\section{Referencias generales}

Las actividades de docencia en el CEG (Centro de Estudios Generales) vinculadas a la temática de envejecimiento y vejez en especial referencia a los Cursos de Cultura y Persona Adulta Mayor, tienen como fondo histórico, según relata León Fernández (2010), los llamados cursos Taller Aula Libre o COCERP, los cuales eran cursos sin créditos que se ofertaban en 1996 en la maya curricular de los CEG de la Universidad Nacional. Estos cursos, se afirma, no tenían créditos; pero eran requisitos para que el estudiantado pudiera graduarse en sus carreras universitarias.

En ese mismo año se formula el curso "El Aporte del Adulto Mayor", el cual inicia la vinculación del estudiantado con las personas adultas mayores que se encontraban en los centros diurnos, hogares o alberges. Las actividades académicas de las aulas se entrelazaron con el trabajo de campo con miniproyectos de alcance comunitario, que atendían algunas necesidades de las personas adultas mayores de varias localidades en el país.

Cuatro años después, en el año 2000, se evalúan estos diálogos intergeneracionales vinculando la docencia con las actividades de campoyla investigación universitaria; profundizando la relevancia de estas actividades en el quehacer académico del CEG; en esta fecha nace el Proyecto de Extensión denominado "Generación Dorada" que, aunado a las labores de docencia, extensión e investigación y otras actividades de producción como la recopilación de historias de vida, abonan las condiciones para la creación del Programa de Atención Integral de la Persona Adulta Mayor (PAIPAM) el cual se adscribe al Centro de Estudios Generales.

Para el año 2011 se generan una serie de nuevas iniciativas entre ellas la formulación y ejecución del Proyecto UNA-VIDA, donde las encargadas de las diversas actividades ya disponían de experiencias y vivencias acumuladas que permitirían conformar un equipo de trabajo que impulsa tres líneas de acciones diferenciadas, las cuales realimentan los propósitos y objetivos de este proyecto.

Entre estos objetivos se encuentra la contribución para ampliar y profundizar el conocimiento sobre los procesos de envejecimiento, abordar el análisis crítico de la vejez desde una visión activa y participativa que favorezca la construcción de proyectos de vida saludables y preventivos. Propiciar diálogos intergeneracionales como otra línea de acción entre estudiantes y personas adultas mayores en centros diurnos y hogares, con miras a la apropiación de una 
nueva visión sobre el envejecimiento y la vejez, destacando la importancia del uso creativo y constructivo del tiempo libre.

El propósito es sensibilizar y generar procesos educativos que promuevan aprendizajes significativos duraderos para la vida. También están la capacitación continua dirigida a las personas adultas mayores, a las personas cuidadoras de este grupo poblacional, las familias, sujetos encargados o responsables de instituciones de cuido y público en general interesado en participar de charlas, conferencias y foros.

El proyecto UNA-VIDA tiene como actores principales a las personas adultas mayores localizadas en centros diurnos y hogares de la provincia de Heredia, las personas adultas mayores procedentes de varios lugares del país que matricularan los cursos que se ofertaban para ellas desde el proyecto; estudiantado universitario procedente de diversas carreras de la universidad que matriculan el curso de cultura y personas adultas mayores, personal académico responsable de las actividades, las personas responsables de los centros diurnos y hogares, los facilitadores y las facilitadoras externas e internas de la UNA para las capacitaciones y las personas de la comunidad nacional interesadas en cursos de capacitación, charlas, conferencias en temáticas variadas referenciadas a temas de envejecimiento y vejez.

Las experiencias y vivencias desde los tres componentes del proyecto han sido enriquecedoras y han aportado significativamente en una nueva mirada y comprensión hacia los temas de envejecimiento y vejez, al tiempo que han permitido repensar, entre otras cosas, las posibilidades que ofrecen los diálogos y los programas intergeneracionales entre personas universitarias y adultas mayores, como herramientas en la construcción de nuevos empoderamientos que conlleven a la construcción de una sociedad más solidaria, respetuosa e inclusiva.

\section{Proyecto UNA-VIDA "Educando para la recreación y uso del tiempo libre construyendo una vejez saludable": Miradas a una experiencia de diálogo intergeneracional vinculando docencia y extensión}

La plataforma estratégica que permite desarrollar estos espacios intergeneracionales es la docencia mediante el Curso Cultura y Persona Adulta Mayor: Retos del Nuevo Milenio; el cual entrelaza los ejes y contenidos temáticos con la formulación de proyectos intergeneracionales diseñados por los equipos de estudiantes, a partir de las visitas de campo realizadas a hogares y centros diurnos de la provincia de Heredia. Algunas de estas instituciones pertenecen a la red 
de la Federación Cruzada Nacional del Anciano (FECRUNAPA) y otras son de carácter independiente producto de la organización comunitaria o por iniciativa de miembros de estas localidades.

Se enfatiza en el vínculo teoría y contacto con la realidad, el curso es un taller donde se debate y comparte sobre temáticas vinculantes con los procesos de envejecimiento y vejez. Algunos de los materiales que se utilizan para desarrollar contenidos programáticos son elaborados por el Consejo Nacional de la Persona Adulta Mayor (CONAPAM), generalmente son revistas, las cuales son analizadas y comentadas en las clases, se busca que estas sean compartidas con las familias por medio de asignaciones vía tareas, ensayos, entre otros, en los espacios de horas de estudio independiente.

La experiencia ha demostrado que estas conectividades redundan en un apoyo directo de las familias a los proyectos que se ejecutan en las comunidades mediante los programas intergeneracionales y contribuyen a una mejor comprensión de los temas de envejecimiento.

Antes de ingresar a los centros diurnos y hogares se realizan una serie de talleres de inducción a estudiantes que permiten, por un lado, orientar las tareas para el diseño de los programas intergeneracionales y, por otra parte, posibilitan compartir normas y reglas coordinadas con las instituciones para el ingreso del estudiantado. Previo a iniciar las visitas de campo se enfatiza sobre los problemas generados por los mitos y los estereotipos construidos sobre las PAM en las sociedades occidentales y se analiza el caso costarricense en particular.

Los ejes temáticos del curso abordan, además, los aspectos socio demográficos, culturales y políticos, también las construcciones teóricas sociológicas sobre el envejecimiento y la vejez, los estilos de vida saludables asociados a factores socioeconómicos y culturales, sobresale la relevancia de los programas intergeneracionales, el envejecimiento activo, las redes formales e informales de atención, la política nacional de envejecimiento y la legislación costarricense en materia de envejecimiento y vejez, la familia y la construcción de lazos de solidaridad y compromiso, entre otros.

En las visitas de campo se elaboran diagnósticos institucionales que dan cuenta del contexto histórico de estas, aspectos relacionados con el personal de apoyo a las condiciones de infraestructura, a las características de la población adulta mayor, 
se indaga sobre las condiciones de salud, lugares de residencia, condiciones de pago, se conversa con las PAM (personas adultas mayores), etc.

Lo anterior permite, por un lado, disponer de una herramienta a considerar en la elaboración de los programas Intergeneracionales orientados a la formulación de actividades recreativas y, por otro, disponer de un" tablero sociocultural" en relación con los centros diurnos y hogares. Estas experiencias rompen con las ideas de homogenización que caracterizan el discurso sobre personas adultas mayores.

En cada ciclo lectivo se abren dos cursos de Cultura y Persona Adulta Mayor donde sus estudiantes diseñan entre 8 y 10 proyectos recreativos acordes con las necesidades y particularidades de las PAM de cada centro y hogar.

Desde el 2011 al 2015 participan al año cuatro grupos de Estudios Generales entre el I y II ciclo lectivo, la composición de cada uno depende de la matrícula. Según los informes presentados y enviados al Sistema de información general (SIA) de la Dirección de Extensión de la Universidad Nacional, hoy Vicerrectoría, para el 2011 fecha de formalización del Proyecto UNA- VIDA ante esta última instancia administrativa- académica (cabe indicar que algunas de estas acciones se habían realizado como actividades conexas al curso pero no con la forma de proyecto) participan aproximadamente 140 estudiantes y 389 PAM, se ejecutaron 16 proyectos recreativos intergeneracionales. En el año 2012, 147 estudiantes y 272 PAM con 16 proyectos recreativos, en 2013, 137 estudiantes y 489 PAM se realizan 9 diálogos intergeneracionales sobre temáticas de alimentación, jubilación, recreación, se ejecutan 17 proyectos recreativos. En el año 2014, 144 estudiantes y 260 PAM se ejecutan 10 proyectos recreativos y 9 diálogos intergeneracionales y para el año 2015 participan 142 estudiantes, 394 PAM y se ejecutan 17 proyectos recreativos. Las labores de coordinación y organización para la participación del estudiantado en estas actividades están a cargo de una docente (la suscrita que escribe este artículo).

Los centros diurnos y hogares participantes en las actividades intergeneracionales recreativas se encuentran en las comunidades de Barva, Santa Bárbara, San Joaquín de Flores, San Antonio de Belén, Santa Rosa de Santo Domingo de Heredia, el Cantón Central de Heredia, los Lagos, centro Lagos la Granada, la Puebla de Heredia, la Aurora de Heredia, San Lorenzo, San Isidro de Heredia, San Juan de Santa Bárbara, entre otros, todos en la provincia de Heredia. 
Los programas intergeneracionales diseñados mediante proyectos con actividades recreativas son evaluados en el aula previamente a su ejecución, con lo cual se garantiza que ninguno de los programas y sus actividades lesionen ni física ni emocionalmente a las PAM; se comparten las propuestas en el aula se exponen y, posterior a la ejecución, se socializan las experiencias.

De manera que los programas intergeneracionales se constituyen en un medio que posibilita el dialogo intergeneracional y la vinculación docencia - extensión y contribuyen, de manera importante, en la construcción de aprendizajes tanto para las personas adultas mayores como para las personas universitarias que participan en el proyecto.

\section{Los programas intergeneracionales como oportunidades y espacios para construir lazos de solidaridad}

Una de las estrategias que se han utilizado para vincular la docencia y la extensión para promover los diálogos intergeneracionales, como se ha mencionado, ha sido la elaboración de programas intergeneracionales (PI) enfatizando la recreación y uso del tiempo libre en proyectos.

Existe una serie de definiciones muy variadas sobre programas intergeneracionales (PI) que Sánchez y Newman (2007, p. 37) retoman de algunos autores entre ellas se destacan las siguientes:

- «Actividades o programas que incrementan la cooperación, la interacción y el intercambio entre personas de diferentes generaciones. Implican compartir habilidades, conocimientos y experiencias entre jóvenes y mayores» (Ventura-Merkel y Lidoff, 1983).

- «... juntan a jóvenes y mayores para compartir experiencias que benefician a ambos grupos. ... Están diseñados para que personas mayores y jóvenes sin lazos biológicos se impliquen en interacciones que promueven los lazos afectivos entre las generaciones y el intercambio cultural y que proporcionan sistemas de apoyo positivo que ayudan a mantener el bienestar y la seguridad de las generaciones jóvenes y mayores» (Newman, 1997).

- «Los programas intergeneracionales son vehículos para el intercambio determinado y continuado de recursos y aprendizaje entre las generaciones más viejas y las más jóvenes para [lograr] beneficios individuales y sociales» (Hatton-Yeo y Ohsako, 2001). 
- «Un programa organizado para fomentar interacciones entre niños, jóvenes y personas mayores que sean continuadas, mutuamente beneficiosas y que conduzcan al desarrollo de relaciones» (McCrea, Weissman y ThorpeBrown, 2004).

- «Actividades o programas que incrementan la cooperación, la interacción y el intercambio entre personas de distintas generaciones. Estas personas comparten sus saberes y recursos y se apoyan mutuamente en relaciones que benefician tanto a los individuos como a su comunidad Estos programas proporcionan oportunidades a las personas, a las familias y a las comunidades para disfrutar y beneficiarse de la riqueza de una sociedad para todas las edades» (Generations United, s.f.).

A pesar de las diferencias conceptuales existen elementos presentes que son un común denominador en ellos. En primer lugar, en todo programa intergeneracional participan personas de distintas edades; en segundo lugar, implican actividades dirigidas para alcanzar fines que sean beneficiosos para todas las personas que participan de él, y tercero, mantienen relaciones de intercambio.

Los PI son medios y herramientas a las cuales se pueden acceder para las gestiones académicas de la docencia y la extensión comunitaria que permiten el diálogo entre generaciones; el compromiso intergeneracional, y pueden incidir en la desmitificación de patrones culturales y políticos enraizados en viejas visiones y concepciones de mundo sobre la juventud y la vejez, al tiempo que abren la posibilidad de compartir para aprehender juntos y crear lazos fuertes de solidaridad intergeneracional.

Estos programas se han posibilitado en la experiencia del CEG por medio de los cursos de cultura y persona adulta mayor como se ha mencionado anteriormente, los cuales han incorporado actividades intergeneracionales recreativas en proyectos de participación comunitaria, y ha estado fuertemente vinculados a los aprendizajes para la vida, reflexionando desde el seno familiar y comunal como nuevas posibilidades para la construcción y fortalecimiento de una sociedad para todas las edades.

\section{Las actividades recreativas en los programas intergeneracionales y los aprendizajes significativos para la vida}

Las actividades intergeneracionales que entrelazan la docencia y la extensión son espacios universitarios potenciales para promover aprendizajes significativos 
para la vida. Sobre este tipo de aprendizaje se hace referencia de manera general a algunos teóricos sin pretender la exhaustividad por las limitaciones propias de este espacio.

Uno de los exponentes relevantes en relación con el aprendizaje significativo ha sido Ausubel, mencionado por Novak (1977) quien señala que es “....un proceso por el que se relaciona nueva información con algún aspecto ya existente en la estructura cognitiva de un individuo y que sea relevante para el material que se intenta aprender".

El aprendizaje significativo permite desarrollar estrategias para resolver problemas, por lo que no permite la acumulación de información sin la comprensión de su significado, así mismo se basa en los principios de diferenciación progresiva y reconciliación integradora. El primer término consiste en presentar primero las ideas más generales y poco a poco, a través del trabajo que conjuntamente realizan docente y estudiantes, se precisan detalles y elementos específicos.

La reconciliación integradora permite encontrar vínculos entre toda la información, relacionar ideas, reconciliar datos que aparentemente no estaban conectados; se trata, entonces, de ver el conocimiento como una unidad y no en forma parcializada. Se enfatiza la importancia de establecer puentes cognitivos, lo cual se logra por medio de organizadores previos, que consiste en buscar términos, ideas o hechos que son familiares para la persona que aprende.

Es importante establecer la diferencia entre aprendizaje memorístico y aprendizaje significativo; el primero recurre exclusivamente a la memoria, a la respuesta mecánica, repetitiva; el segundo tiene lugar cuando el aprendizaje o cuando la experiencia de aprender tiene sentido y significado para el alumnado.

El aprendizaje memorístico no organiza el material que se aprende como una unidad, se dan datos que no se relacionan con los conocimientos previos del estudiantado; por consiguiente, no se hacen relaciones entre conceptos ni se articulan, por lo que el alumnado debe esforzarse por aprender mecánicamente.

Desde esta concepción del aprendizaje, el alumnado ejercita su memoria a corto plazo; pero al no darse una verdadera comprensión, análisis y funcionalidad de los elementos que "grava" existe una alta posibilidad que estos conocimientos sean olvidados en un lapso relativamente corto. Cabe anotar, que el aprendizaje memorístico favorece la acumulación de la información, pero no facilita el 
desarrollo conceptual ni la expansión del conocimiento, pues, según Novak (1977), en este tipo de aprendizaje la información nueva no se asocia con los conceptos existentes en la estructura cognitiva y, por lo tanto, se produce una interacción mínima o nula entre la información recientemente adquirida y la información ya almacenada o que ya se poseía.

Contrariamente, el aprendizaje significativo relaciona los nuevos conceptos con elementos ya existentes en la estructura cognitiva, por lo que los conceptos están articulados en unidades que tienen sentido para el estudiantado; facilitan la comprensión y logran establecer conexiones entre conceptos más generales y con mayor poder explicativo a los más específicos, la información tiene sentido en su totalidad y evita la desarticulación de conceptos.

Por esto, los espacios propiciados desde el curso y la extensión se han constituido en medios y posibilidades para acercar a las personas jóvenes y a las personas adultas mayores; repensarnos como personas humanas que compartimos, a pesar de las brechas generacionales; compartir diálogos y saberes, el deseo de disfrutar, de reír, de pensar, de estar juntos y juntas; de visualizar las potencialidades, necesidades, intereses de "las otras y los otros" y, por supuesto, de aprehender, reaprehender y desaprehender conjuntamente y de manera recíproca.

La elaboración de los programas recreativos entrelazándolos con las necesidades y requerimientos de las personas adultas mayores y estas con los retos y desafíos de las actividades que se programan y ejecutan son espacios potencializadores que estimulan este tipo de aprendizaje intergeneracional significativo. En el ciclo vital de la vida por el cual todas las personas transitamos, estamos en conectividades entre conocimiento existente y nuevo conocimiento y entre estos y nuevas experiencias construidas y cercanas a nuestros entornos, por las características mismas del curso y la temática de envejecimiento y vejez, los aprendizajes significativos son referenciales a la vida, a nuestro hacer y hacernos cada día.

Se entrelaza teoría y práctica, se elaboran y analizan propuestas de programas recreativos que incluyen diálogos intergeneracionales, se ejecuta y evalúa lo planeado y se sistematizan las experiencias; todo en conjunto estimula y propicia aprendizaje significativo que se pretende sea duradero para la vida.

El "vehículo" sobre el cual se construye los programas intergeneracionales y, por ende, el dialogo intergeneracional, son las actividades recreativas, las cuales 
tienen una relevancia significativa para el desarrollo social, psicoemocial y cultural de las personas. El ocio, el disfrute del tiempo libre como una de las necesidades básicas, abre la posibilidad de encuentros como una vía para el aprendizaje intergeneracional significativo.

Se asume la recreación como mediadora de procesos de desarrollo humano, lo cual significa comprenderla desde sus beneficios en cada una de las dimensiones o componentes de desarrollo de los seres humanos y considerando las condiciones que hacen posible estos posibles beneficios.

Las prácticas recreativas puedensercomprendidascomoherramientasquecomplementan los procesos de aprendizaje y desarrollo cultural y, como tales, proporcionan un medio para estimular el crecimiento personal y grupal de manera positiva. La recreación orientada al desarrollo de las capacidades, habilidades y destrezas es un satisfactor de las necesidades humanas de autonomía, expresión personal e interrelación social dentro de los diversos entornos socioculturales y políticos.

Entenderemos como recreación lo anotado en la Ley 181, artículo 5, de 1995 en Colombia, tal y como lo cita Wiswell:

Un proceso de acción participativa y dinámica que facilita entender la vida como una vivencia de disfrute, creación y libertad, en el pleno desarrollo de las potencialidades del ser humano para su realización y mejoramiento de calidad de vida individual y social, mediante actividades físicas o intelectuales de esparcimiento (2009).

Sobresalen, en este sentido, algunas características como la espontaneidad, alegría, regocijo disfrute, libertad, creatividad, socialización y goce personal Romero (2008). En las experiencias que se comparten aquí, de conformidad con lo señalado, se trata de actividades educacionales o pedagógicas, que son aquellas donde la persona adulta mayor y la persona joven se incorporan en el proceso de enseñanzaaprendizaje, ayudándoles a incrementar su formación individual y grupal, mediante la reeducación y aprendizajes de nuevos conocimientos y experiencias.

Se apunta en estos programas intergeneracionales a una recreación formativa para todas las edades, ya que si bien es cierto como señala Alzate:

Desde sus inicios la recreación ha sido considerada como un alivio a las condiciones de cansancio y aburrimiento, este es un esquema de compensación de 
algún problema o situación del ser humano desde este enfoque "compensatorio" la recreación ha sido visualizada como experiencias de entretenimiento y diversión suplidoras de necesidades del momento, con lo que se ha convertido en un fin más que en un medio para el desarrollo pleno de las personas (2002).

Por lo que se coincide, en este artículo, con lo señalado por Alzate donde se supera esa visión tradicional:

En los últimos años se ha venido dando importancia a los contenidos didácticos de los programas, aportando a los participantes enseñanzas por medio de la recreación como dinamizador de procesos, lo cual ha permitido que esta actividad contribuya de una mejor manera con la formación y el desarrollo integral de las personas que viven este tipo de experiencias. Esta tendencia se ha llamado "Recreación Formativa" y en este momento significa una opción adecuada para permitir que la recreación tenga un propósito significativo (2002).

Se asume, entonces, que la recreación tiene un valor educativo, tal y como lo apunta Romero (2008), por las siguientes razones:

- Establece un conjunto de valores que contribuyen a la formación de la persona.

- Permite el desarrollo integral de la persona.

- Permite la transmisión de la herencia cultural.

- Fomenta el uso constructivo del tiempo libre.

- Adapta socialmente a la persona.

- Despierta la sensibilidad social.

- Fomenta el bienestar y la proyección personal y social.

- Estimula el desarrollo de actitudes positivas.

- Fortalece sentimientos de fe, solidaridad, confianza, optimismo, deseo de superación y autorrealización.

- Rescata y fortalece valores culturales de nuestra nación.

- Induce nuevos hábitos de utilización del tiempo libre.

- Estimula el desarrollo de la expresión de los sentimientos y las emociones. 
- Propicia el descubre, reconocimiento y desarrollo de todo el potencial creativo.

- Satisface necesidades de orden espiritual y estético.

- Promueve oportunidades para desarrollarse integralmente.

En muchas ocasiones se tiende a ver a las personas adultas mayores únicamente como receptoras de los bienes y servicios prestados por la generación "laboralmente activa". En el ámbito familiar se produce un flujo de intercambios que va en las dos direcciones: la generación adulta de hijos e hijas, al tiempo que provee a sus padres y madres de apoyo material y de afecto, recibe también de ellos y ellas ayuda y afecto (Bazo, 2002).

Son diversos los tipos de intercambio que tienen lugar entre las distintas generaciones, algunos de carácter instrumental y otros de carácter afectivo. Suele pensarse que son las personas jóvenes las únicas que realizan aportaciones, cuando la realidad es que las personas mayores contribuyen de forma considerable al bienestar familiar e incluso social (Bazo, 2003).

Tomando en consideración este marco, los programas intergeneracionales son vehículos para el intercambio determinado y continuado de recursos y aprendizaje entre las generaciones más viejas y más jóvenes para beneficios individuales y sociales. Según Hatton y Ohsako (2001), los intercambios intergeneracionales son un instrumento para la solución de problemas sociales prioritarios de un país, dado que unen generaciones separadas, rescatan y replantean la transmisión de la cultura tradicional, fomentan una vida laboral y social activa entre generaciones y se comparten recursos entre las generaciones.

Los intercambios intergeneracionales, por su naturaleza, proporcionan un marco para el desarrollo de un enfoque coherente del aprendizaje significativo de toda la vida, el cual está firmemente arraigado en la educación comunitaria. Estos reconocen que el aprendizaje es un proceso que ocurre durante el curso de la vida, que las personas pueden tener necesidades e intereses diferentes en distintas fases de sus carreras y brindan el potencial para el aprendizaje compartido, a través de las generaciones para enriquecer el plan de estudios para todos y todas.

La recreación, el uso del tiempo libre y la educación para las personas adultas mayores y jóvenes: Disfrutando el tiempo libre y construyendo proyectos alternativos de vida mediante aprendizajes significativos. 
El disfrute adecuado del ocio es estimular la imaginación, dar rienda suelta a la fantasía, potenciar nuestras actividades manuales, favorecer la capacidad de creación que existe en todas las personas, experimentar, asumir retos, sentirnos a gusto e ir moldeando nuestro carácter y nuestra personalidad de forma equilibrada y armónica.

Según Urbano, Yuniy Tarditti (2002), la educación de mayores puede caracterizarse como un conjunto de acciones intencionales mediante las cuales se produce un proceso de reconfiguración de la identidad personal. En otras palabras, la acción educativa promueve el aprendizaje, entendido este no como algo externo que se incorpora al sujeto (conocimientos), sino como un proceso de reconfiguración interna que le ocurre y atraviesa y que está directamente ligado a la experiencia, más que a la información.

Desde esta perspectiva, el concepto de recreación adquiere otro significado y puede articularse como una herramienta teórica que permita una mejor comprensión de los alcances y sentidos que la educación puede adquirir en esta instancia del ciclo vital de la vida.

Para Ballestero (2001), crear nuevas oportunidades para usar de manera creativa y constructiva el tiempo libre es esencial en las personas adultas mayores, a fin de que los individuos mediante el proceso de envejecimiento disfruten de plena y adecuadamente de este mismo. De manera que, al entrar al periodo de jubilación, se tenga a mano un buen número de actividades y oportunidades para hacer compartir, investigar, crear y fomentar, en vez de aislarse y contribuir al deterioro individual y familiar.

Tomar decisiones de qué hacer en el tiempo libre implica también recrear la propia dimensión subjetiva, ya que implica, entre otras cosas, sostener el deseo propio frente a los otros individuos y enfrentar el desafío de apartarse de los roles establecidos (estatuidos) en acuerdo explícito o implícito con otros miembros de la constelación familiar: cónyuge, hijos e hijas, hermanos y hermanas, nietos y nietas.

Según Urbano (2002), recrear la mirada acerca de la vejez supone cuestionar las teorías que subyacen a nuestras prácticas a la luz de los efectos que estas producen, los cuales deben ser contrastados en la experiencia misma y en el discurso de la población adulta mayor que circula en estos lugares de recreación. Solo "recreando" la mirada sobre la vejez estos lugares tendrán una eficacia simbólica 
positiva, en donde se trabaje con los aspectos saludables que promueven la autonomía y que refuerzan los recursos con que cuenta el sujeto.

Por eso es necesario reeducar para la utilización del tiempo libre, el ocio y la recreación no solamente a las personas adultas mayores, sino también y en interrelación, a las personas jóvenes, para que estas tengan las herramientas necesarias para aprovechar de una mejor manera su tiempo, realizando actividades confortables que satisfagan las necesidades personales, las cuales van a llevar al crecimiento personal.

Retomando, la recreación en relación con los proyectos formulados y ejecutados por medio del curso cultura y persona adulta mayor se sustentan mediante el contacto personal del estudiantado con las personas adultas mayores en los diferentes centros diurnos y hogares de algunos lugares de la provincia de Heredia.

En primer lugar, se elaboran diagnósticos que permiten disponer de información pertinente y oportuna para la planificación y ejecución de las diversas actividades recreativas, además de otras informaciones que posibilitan la elaboración de menús para meriendas y almuerzos, elaboración de materiales e implementos.

Los proyectos son presentados en un primer momento como propuestas con la finalidad de ser analizados en el aula, los cuales una vez que hayan incorporado las observaciones se ejecutarán en los distintos lugares. Este procedimiento se realiza para garantizar la integridad física-emocional de las personas adultas mayores en los centros y hogares, y el ajuste de su pertinencia a las condiciones tanto socio-espaciales de las instituciones, a las características y requerimientos de las personas adultas mayores y a las condiciones y posibilidades del estudiantado.

Lo anterior ha sido una garantía brindada a las diversas instituciones y una responsabilidad asumida directamente por la docente a cargo. De tal manera que se debe cuidar que la implementación de los proyectos cumpla con las normas establecidas en cada una de las instituciones, en seguridad personal, disciplina, orden, aseo y respeto, entre otros; por lo cual al estudiantado también se les capacita en este sentido.

Cabe mencionar que las actividades a ejecutar son consensuadas entre los grupos de personas adultas mayores y de estudiantes; esto es muy importante porque, por un lado, se requiere que las personas adultas mayores disfruten de ellas y, por otro lado, que sean posibles de llevar a cabo con los recursos disponibles y 
capacidades para su elaboración por parte del estudiantado. Un ejemplo de esto último son aquellas actividades que requieren elaboración propia de materiales como balones, bolos, tableros especiales, implementos varios adaptados a las necesidades de las personas adultas mayores con alguna necesidad física o educativa especial.

Las experiencias en el aula, tanto antes como después de la ejecución de los proyectos, han sido muy enriquecedoras porque permite, entre otros aspectos, compartir vivencias, experiencias, momentos muy significativos, esfuerzos realizados. También permiten visualizar y evidenciar los diversos escenarios socioculturales, socioeconómicos y la condición física y psicoemocional de las personas adultas mayores, desmitificando la pretensión de que todas tienen las mismas situaciones y condiciones.

De los aprendizajes significativos- programas y actividades recreativas: Algunas experiencias narradas por estudiantes participantes en programas intergeneracionales en el curso Cultura y Persona Adulta Mayor

Como parte de las actividades intergeneracionales (a lo largo de cinco años del 2011 al 2015) el estudiantado escribe su experiencia y la comparte con el grupo. En este artículo se socializan algunas representativas de los cientos de experiencias narradas y contadas en el curso. Por el espacio se mencionan solo algunas de ellas, selección difícil, pero son una muestra. No se dispone de registro de parte de las personas adultas mayores, pero igualmente las experiencias han sido muy satisfactorias.

Yassiny Salas “...Para mí esta experiencia ha sido excelente, compartir con ellos y no me arrepiento de haberlo hecho. Este curso nos ha enseñado el valor y el verdadero sentir de la vida yo en lo personal aprendí mucho. Que hay que cuidarse cuando se es joven para tener una vejez saludable, el envejecimiento es una etapa linda no como se tiene en concepto que es lleno de enfermedades y todo el montón de mitos que se les señalan. Para mí esta es una experiencia que volvería a repetir sin pensarlo estas personas son maltratadas muchas veces pero la gente no se da cuenta del gran valor que tiene ese carisma que tiene guardado para dar y recibir".

Darling Sánchez Ríos "En conclusión fue una maravillosa experiencia llena de muchas emociones donde Dios el cariño la felicidad y diversión estuvieron presentes. De verdad que debemos valorar a estas lindas personas y deberíamos 
sacar un poco más de tiempo para compartir con todos ellos... No hay las suficientes palabras para describir todo lo sentido ni el tiempo para contar todo lo vivido. Lo más importante es que esta linda experiencia queda gravada en nuestro corazón y que nunca la vamos a olvidar que cada recuerdo va a permanecer siempre ahí."

Brenda Herrera Quirós "La oportunidad que me dio este curso y la profesora Ana Monge de compartir y crear un diálogo intergeneracional con los adultos mayores, de una forma dinámica y sensibilizadora, fue una experiencia inolvidable y enriquecedora, ya que me permitió abrir los ojos y dejar de lado los mitos y prejuicios que tenía sobre las personas adultas mayores... Asimismo, los momentos que disfrutamos en el Centro Diurno de Santa Bárbara de Heredia, fue el claro ejemplo que cuando uno quiere disfrutar y ganas de vivir lo hace a pesar de todo, en el centro encontramos diferentes características en los adultos mayores como: los más alegres, los que han sufrido grandes pérdidas, los artísticos... cada uno de ellos tiene una historia de vida, que mejoró y contribuyó a cambiar la nuestra..."

Allan Montero Vizcaíno "Para mí ha sido de gran valor haber podido vivir de esta experiencia tan hermosa como lo fue trabajar con las personas adultas mayores de esta localidad gracias a este curso me pude sensibilizar a las necesidades y deseos de estas personas a estar anuente de que todos vamos por el mismo camino y en algún momento tenemos que envejecer, tal vez antes yo no era un joven con gerontofobia pero si era una persona que no tenía tan presente las necesidades de estos sectores de la población que realmente son de mucho provecho a la sociedad estas personas han dado mucho, ellos han sido los que forjaron los caminos por los que va este país, gracias a ellos lo bueno que somos ha sido por ellos donde sea que veamos hay un adulto mayor nuestro padre o madre, abuelo(a), tío(a), vecino, maestros, etc. Y ellos tienen necesidades de las cuales nosotros no podemos omitir debemos apoyarlo"

Katherine Solórzano García. "El trabajo que hemos realizado me parece verdaderamente bello, ya que nos ayudó a sensibilizarnos de corazón hacia estas personas, ya que a veces nos cuesta un poco relacionarnos y comprenderlos.

Al ver este curso en el monitor de la computadora lo vi muy interesante porque sabía que me iba a enseñar muchas cosas, ya que mis padres son los futuros adultos mayores y entenderlos, para mí en esa edad sería algo muy agradable, ya que ellos siempre tratan de entenderlo a uno; lo que jamás espere es que nos 
iban a enseñar eso estando personalmente con este tipo de personas, lo cual me llamo aún más la atención. Sinceramente este curso me enseño bastante en todo el ámbito de la PAM y le doy gracias a Dios, a la profesora y a los adultos mayores ya que gracias ellos pude experimentar este agradable momento y también gracias a su trato, cariño y experiencia me enseñaron demasiado; ahora los podré entender mejor y tenerles paciencia ya que antes tenía otro tipo de mentalidad y gracias a ellos la logré modificar, ahora podré sentarme hablar y a escuchar a mis abuelitos, padres y a cualquier adulto mayor."

Santiago Badilla Vega "Durante todo el curso cultura y adulto mayor, he sido parte de un continuo proceso de crecimiento y sensibilización sobre los adultos mayores. He comprendido que los adultos mayores son una población que es constantemente discriminada, simplemente porque no posee la misma edad que tienen los demás. Las actividades realizadas nos hicieron crecer como personas y como estudiantes, sin contar el nivel de sensibilización que recibimos a lo largo del curso; pudimos comprobar cómo los adultos mayores a pesar de sus limitaciones físicas querían participar en todas las actividades, por lo tanto, hicimos un gran esfuerzo por adecuar todo lo que teníamos planeado para que fueran actividades inclusivas. El curso "Cultura y Adulto Mayor" es de mucha importancia, puesto que acciones como esta hacen que la población adulta mayor logre una mayor inclusión en la sociedad, que ya no se vean como si fueran otro tipo de ser humano, que se logren desarmar los mitos que rodean a esta población. Es importante que se sigan dando estos tipos de sensibilización no solo en la Universidad, sino en colegios y en escuelas donde se logre inculcar el respeto y la inclusión.

María José Bolaños León “ Por medio de este curso pude vivir experiencias únicas e inolvidables que no las cambiaría por nada, en mi opinión es un curso completamente diferente a los demás que brinda la Universidad Nacional porque además de teoría se ve un mayor esfuerzo de reflejar la parte humanitaria de nosotros(as) los(as) estudiantes aplicada en una acción social donde logramos incluir e interactuar con las PAM en nuestras actividades diarias, buscando así tomar consciencia y hacer un cambio en una sociedad tan excluyente social, física y laboralmente, en especial a estas personas que ya van entrando a la etapa de la vejez; todo esto es muy importante..."

Ana Graciela Haug Cordero "Al recibir este curso aprendí mucho sobre la persona adulta mayor, conceptos y teorías que desconocía; pero al llegar al 
centro con el programa intergeneracional amplié mi percepción sobre ellos, son personas que necesitan atención y que alguien dedique un tiempo para compartir para comentar sencillas anécdotas o acontecimientos impactantes que han vivido. El lapso que pase en este lugar fue de crecimiento propio y no por pasar una materia más, sino lo consideraba como desestresante ir a compartir y estar un rato con ellos ".

Yerling Sibaja Elizondo "Mi experiencia en el taller recreativo para mí fue muy enriquecedora, el hablar, bailar, escuchar sus anécdotas; el simple hecho de compartir con ellos es algo muy satisfactorio, ya que son personas con tanta experiencia con tanta alegría y vitalidad, con tanto amor que darnos es la mejor experiencia que he tenido en mi tiempo en la universidad..."

Jordy Araya Ramírez "Verdaderamente el trabajo realizado es gratificante, y en gran manera sensibiliza el corazón humano. El trabajar con este tipo de población puede ser muy influyente en el acontecer de nuestras vidas de ahora en adelante.

Al matricular el curso detrás de una computadora puede ser realmente sencillo, y las expectativas no van más allá de lo normal; pero es grandioso toparse con este tipo de actividades en transcurso del curso. Ahí es donde todo lo aprendido en las lecciones y en las lecturas se pone en manifiesto".

Kevin Azofeira Camareno "En este proyecto aprendí muchas cosas sobre los adultos mayores, la experiencia en el centro diurno que nos tocó fue muy enriquecedora y gratificante. El estar compartiendo con cada una de las personas adultas mayores del centro diurno de San Joaquín de Flores me dio la visión de las diferentes situaciones que pasan ellos cada día y de esta forma poder entenderlos mejor.

Todo lo que compartimos con ellos me ayudo pienso yo, a crecer más como persona, a pensar de forma diferente..."

Laura Miranda "Llevar este curso me sirvió mucho en la parte personal porque a través de él pude interiorizar, tener nuevas ideas y significados del proceso de envejecimiento. Gracias a todo ello, en estos momentos puedo tener una concepción distinta de ellos a las que tiempo atrás tenía, puedo convivir más fácilmente, tener el respeto y actitud suficiente para hacerlo. 
En esta experiencia de visitar la Asociación Pro Adulta Mayor de los Lagos pude poner en práctica todo lo aprendido".

$\mathbf{M}^{\mathbf{a}}$ Laura Elizondo "Este curso me deja una experiencia maravillosa, ya que nos enseñó muchas cosas de las personas adultas mayores que antes no sabíamos o que simplemente ignorábamos y dejábamos pasar por falsas imágenes que nos presentaba la sociedad. El compartir con las personas adultas mayores es algo que deja una gran huella en mí, porque hay tantas cosas que aprender de ellos, sobre sus experiencias de vida, su alegría, el poder relacionarnos con ellos para borrar esos estereotipos que tenemos, así también los que ellos tienen de nosotros".

\section{Reflexiones finales}

Los proyectos universitarios que vinculan la docencia con la extensión en actividades intergeneracionales, como los desarrollados en el proyecto UNA Vida por medio programas recreativos, son una simbiosis que generan espacios potencializadores y oportunidades para una deconstrucción individual y colectiva del significado y percepción sobre los procesos de envejecimiento y vejez.

Los programas intergeneracionales engarzados a proyectos de extensión comunitaria acompañados con cursos académicos se convierten en herramientas pedagógicas que permiten aprendizajes significativos para la vida, como se ha evidenciado en esta experiencia académica.

Los proyectos de este tipo abre un abanico de posibilidades en relación con la vinculación docencia -extensión; por supuesto que han existido algunas debilidades y dificultades que tienen que mejorarse como cualquier proceso que inicia, pero los resultados y beneficios obtenidos demandan esfuerzos importantes para superarlos. El nuevo humanismo de hoy requiere superar brechas y estrechar esfuerzos institucionales para la consecución de una educación vinculante con los grandes problemas y retos de nuestro tiempo, que las nuevas generaciones visualicen nuevos espacios y horizontes, nuevas formas de entender y hacerse en el mundo contemporáneo que reclama la emergencia de nuevas respuestas a viejos problemas. Todos los esfuerzos en esta línea redundarán en grandes beneficios para todos y todas, el nuevo humanismo del siglo XXI así lo reclama. 


\section{Referencias}

Alzate, C. (2000). Recreación interactiva: El futuro del uso del tiempo libre. Caja Colombiana de Subsidio Familiar (COLSUBSIDIO). VI Congreso Nacional de Recreación. Vicepresidencia de la República/ Coldeportes/ FUNLIBRE. 10 al 12 de agosto de 2000, Bogotá, D.C. Colombia. Recuperado de http:// www.redcreacion.org/documentos/congreso6/CAlzate.htm

Ballestero, C. (2001). Recreación en el adulto mayor. Revista Anales en Gerontología 3(3), 111-116.

Bazo, M. (2002). Dar y recibir: Análisis comparativo de las prácticas de intercambio entre las generaciones, preferencias y valores en las familias españolas. Revista universitaria de formación del profesorado, 45, 55-65.

Bazo, M. (2003). Intercambios familiares entre las generaciones y ambivalencia: Una perspectiva internacional comparada. Recuperado de http://www. fes-web.org/publicaciones/res/archivos/res02/07.pdf

Borbón, M. y León, M. (2012). Relaciones intergeneracionales: Un encuentro de saberes y solidaridad. Recuperado de http://fiapam.org/wp-content/ uploads/2012/11/RELACI1.pdf

Gutiérrez S. M. y Hernández, H. D. (2013) Revista interuniversitaria pedagogía social. [1139-1723 (2013) 21, 213-235] tercera época. Recuperado de http:// www.upo.es/revistas/index.php/pedagogia_social/article/view/737/568

Hatton, A. y Ohsako, T. (2001). Programas intergeneracionales: Política pública e implicaciones de la investigación: Una perspectiva internacional. Recuperado de http://www.unesco.org/education/uie/pdf/intergenspa.pdf.

León, F.M. (2010). El Programa de Atención Integral de la Persona Adulta Mayor (PAIPAM) de la Universidad Nacional (UNA). Revista Digital Palabras Mayores, 2(4). Recuperado de http://repositorio.pucp.edu.pe/index/bitstream/ handle/123456789/21343/leon\%20fernandez.pdf?sequence=1\&isAllowed=y

León, F. M. (2011). Programa de Atención Integral de la Persona Adulta Mayor (PAIPAM). Universidad en diálogo. Revista de extensión, 1(1). Recuperado 
de http://www.revistas.una.ac.cr/index.php/dialogo/article/view/1223

Neuman, S. y Sánchez M. (2007). Programas intergeneracionales. Hacia una sociedad para todas las edades. Colección Estudios Sociales, 23. Barcelona España Recuperado de http://www.aepumayores.org/sites/default/files/Programas_Intergeneracionales_Coleccion_Estudios_Sociales_vol23_es.pdf

Novak, J. (1982). Teoría y práctica de la educación. Madrid: Editorial Alianza.

Romero Ibáñez, P. (2008). La lúdica y la recreación como proceso pedagógico. Programa de Formación Permanente a Docentes. Recuperado de http://3congresodeeducacion.blogspot.com/2008/10/la-ldica-y-la-recreacin-como-proceso.html

Salazar, C. (2004). Actividades intergeneracionales: Una opción creativa más para las personas adultas mayores. Revista Anales en Gerontología, 4, 29-44.

Sánchez, M., y Mateo, A. (2011). Relaciones intergeneracionales. Envejecimiento activo. Madrid: IMSERSO.

Urbano, C., Yuni, J. y Tarditti, L. (2002). El sentido recreativo de las experiencias educativas con adultos mayores. Recuperado de http://red-am.com.ar/buscador/files/EDUCA028_Tarditti.pdf.

Wiswell Arévalo, M. del A. (2009). Impacto de la recreación en la construcción de procesos de ciudadania: Reflexiones acerca de la relación entre recreación y productividad. Fundación Colombiana de Tiempo Libre y Recreación (FUNLIBRE). Recuperado de http://www.redcreacion.org/articulos/awiswell.html

Vega, J. L. (1992). Las relaciones entre generaciones. España, Badajoz: Infad.

Vigotsky, L. (1979). El desarrollo de los procesos psicológicos superiores. Barcelona: Editorial Crítica. 